\title{
Correction to: Vitamin C mesotherapy versus topical application for gingival hyperpigmentation: a clinical and histopathological study
}

\author{
Mohamed El-Mofty ${ }^{1,2} \cdot$ Sarah Elkot ${ }^{3} \cdot$ Amira Ghoneim $^{4} \cdot$ Dalia Yossri $^{1} \cdot$ Ola Mohammed Ezzatt $^{1}$ (D)
}

Published online: 2 August 2021

(c) Springer-Verlag GmbH Germany, part of Springer Nature 2021

\section{Correction to: Clinical Oral Investigations https://doi.org/10.1007/s00784-021-03978-6}

In the "Treatment protocol and interventions" section, the authors made the description more clinically applicable and refined the description to eliminate any conflict to the practitioners during practicing the technique. The information in reference 32 was also modified to coincide with the reference citation.

The original article has been corrected.

Publisher's note Springer Nature remains neutral with regard to jurisdictional claims in published maps and institutional affiliations.

The original article can be found online at https://doi.org/10.1007/ s00784-021-03978-6.

Ola Mohammed Ezzatt

dr.ola@dent.asu.edu.eg

1 Department of Oral Medicine, Periodontology Oral Diagnosis and Radiology, Faculty of Dentistry, AinShams University, 20 Organization of African Union St., Cairo 1156, Egypt

2 Department of Oral Medicine, Periodontology, Faculty of Dentistry, Nahda University, Beni Suef, Egypt

3 Department of Oral Medicine, Periodontology, Faculty of Oral and Dental Medicine, Future University in Egypt, Cairo, Egypt

4 Department of Pharmaceutics and Pharmaceutical Technology, Faculty of Pharmaceutical Sciences and Pharmaceutical Industries, Future University in Egypt, Cairo, Egypt 